\title{
LA INNOVACIÓN AFECTA LA ESTRUCTURA DE LAS ORGANIZACIONES
}

\section{THE INNOVATION AFFECTS THE STRUCTURE OF ORGANIZATIONS}

\author{
Javier Benalcázar
}

\section{Resumen}

La innovación está fuertemente identificada con el cambio y la creatividad para hacer cosas nuevas, ocasionando transformar y afectar cualquier tipo de industrias o empresas. Por esto es fundamental que las organizaciones mejoren su modelo de negocios aplicando la innovación como parte de sus estrategias, no solo para crear o mejorar un producto, sino también para cambiar la estructura de la organización y así aumentar sus posibilidades de éxito. El presente trabajo recopila importante literatura sobre los tipos de innovación, proceso de cambio (Modelo de Lewin). Este modelo permite entender la necesidad de enfrentar los cambios y, lo importante, de transformarse para evitar perder posicionamiento en el mercado. Finalmente, se realizó un análisis exploratorio a la empresa ecuatoriana Válvulas del Pacifico Pacifvalvs S. A. la cual está inmersa en la industria petrolera. Se analizó los cambios que ha sufrido esta empresa desde que empezó sus operaciones hace 9 años y cuáles han sido sus innovaciones para seguir siendo productiva y rentable.

\section{Palabras claves}

Innovación, incremental, radical, cambio

\section{Abstract}

Innovation is strongly identified with "changes" and "creativity" to do new things, causing to transform and affect any type of industries and/ or companies, therefore it is essential that organizations improve their business model by applying innovation as part of their strategies, not only to create or improve a product but also to change their structure, and thus increase their change of success.

The work collected important literature on the types of innovation, change process (Lewin Model). Follow-up model to be able to understand the need to face changes and the importance of transforming and avoid to losing positions in the market.

Finally, the Ecuadorian company Válvulas del Pacifico Pacifvalvs S.A was analyzed to which it is immersed in the oil industry to observe the changes it has undergone since it started its operations 9 years ago and which were its innovations to continue being productive and profitable.

\section{Keyword}

Innovation; innovation; radical, changing 


\section{Introducción}

Actualmente vivimos en un mundo donde los cambios son más continuos que nunca antes, por lo que las compañías siempre deben buscar estrategias que les permita continuar expandiéndose y creciendo. Las estrategias y decisiones que se materializan dentro de las empresas en el pasado que generaron rentabilidad, no garantizan que tengan el mismo efecto en el presente y futuro de las organizaciones.

Ser innovador es una herramienta importante que mantiene a las personas y las empresas por delante de los competidores. La innovación, a través del tiempo, ha creado riqueza y crecimiento económico. Shumpeter (1911) señaló que la innovación abarca crear nuevos productos o procesos de producción o mejorarlos, lo que puede generar un cambio en los costos de estructura de una industria.

La innovación no es solo crear un nuevo producto o servicio, es también crear un proceso que genere algo nuevo, y que, además, genere valor para él (Wickham, 2006, p. 7). Adicionalmente, Carrol (2009) indica que la palabra innovación proviene del latín root innovatus que significa renovar. Para ser innovadores, es necesario romper la rutina de los procedimientos anteriores.

Las empresas invierten en investigación y desarrollo $(1+D)$, activos, adquisición de nueva tecnología y contratación de personas especializadas. No obstante, estas inversiones no garantizan un futuro cierto de ingresos, rentabilidad y sobrevivencia para las organizaciones (Amit y Zott, 2010).

Los nuevos productos provienen de empresas que se renuevan y de los nuevos competidores en el mercado. Mintzberg in Burn (2001, p. 49) argumenta que no es seguro que el cliente acepte comprar un producto simplemente porque es nuevo. El éxito se encontrará en hacer las cosas de manera completamente diferente. Además, señala que "las innovaciones deben romper el molde de cómo se hacen las cosas". Es decir, las organizaciones deben buscar nuevas formas, mecanismos y procesos de producir y ofrecer sus productos (innovación), si desean satisfacer las necesidades de sus clientes.

Actualmente los clientes son más exigentes en términos de personalización, calidad, novedad y precio. Todos estamos comprometidos con cambios drásticos o pequeños, porque en la vida no hay crecimiento sin cambio. Si las personas siguen haciendo lo mismo en un mercado competitivo como en la actualidad, abren las puertas a nuevos competidores, ponen en riesgo su posición en el mercado, ocasionan disminución de ventas y rentabilidad colocando a las organizaciones en un alto riesgo de supervivencia.

\section{Innovación}

El padre de la innovación es Joseph Schumpeter (Sydie y Adams, 2001), quien se centra más en el desarrollo económico y describió la innovación en cinco tipos en Shumpeter (1934, p. 34):

- Implementación de algo nuevo o mejorado.

- Introducción de un nuevo proceso.

- Creación de un nuevo mercado.

- Identificación de nuevas fuentes de suministro de materias primas.

- Creación de nuevas estructuras dentro de la organización.

Por otra parte, Sundbo (1998, p. 21) señala que la innovación no necesariamente tiene que centrarse solo en el desarrollo económico, sino que también puede tener diferentes caracteres como tecnológico (objetos), comportamiento (estructura de la nueva empresa), movimientos físicos (nuevo transporte) o intelectual (consultoría). 
Por otro lado, Drucker (1985, p. 17) define la innovación como "las herramientas específicas de los empresarios, los medios por los cuales explotan el cambio como una oportunidad para un negocio o un servicio diferente". En otras palabras, Drucker ve el cambio (innovación) como una oportunidad para crear un negocio y así generar riqueza. Hoy en día, el cambio es inevitable; así como, el ambiente empresarial es dinámico, la gente cambia y cada vez es más exigente a la hora de cubrir sus necesidades.

La innovación es importante para lograr una ventaja competitiva en un entorno cambiante. (Dobson, et.al 2004). EasyJet Aerolínea logró un gran éxito cuando la desregulación (política) del gobierno quien permitió a las aerolíneas de bajo costo operar realizando servicios aéreos "sin lujos". Los pasajeros pudieron utilizar la aerolínea sin tener que pagar por la comida o bebidas lo que redujo los costos aéreos, además al permitir a los pasajeros hacer reservas en Internet sin necesidad de imprimir los boletos logro reducir costos de servicios y presentarse como una empresa con responsabilidad ambiental (Palmer y Hartley 2006).

\subsection{Tipos de innovación}

Hay dos tipos de cambios, incrementales y radicales. Para entenderlos es necesario distinguirlos unos de otros. James March (1991, en Leifer et al, 2000, p.4) afirma que el cambio incremental es el uso de la tecnología existente, para obtener mejoras de costos, incrementar beneficios o características de los productos, y mejorar procesos o servicios existentes. Price (2009, p. 13) indica que los cambios incrementales están en una escala menor. Las personas están capacitadas y están apoyadas para contribuir al proceso de cambio. El personal está consciente del porqué de los cambios y porque existen nuevos objetivos. Trabajan para implementar el proceso hasta que se logren las nuevas metas. El cambio incremental no implica la previsibilidad suficiente para estar absolutamente seguro de la magnitud, la frecuencia y la duración de los cambios. El cambio incremental se origina con pequeñas modificaciones en productos o procesos que mejoran la funcionalidad y beneficios del producto o del servicio. Este tipo de cambio constituye la base de la mejora continua y del progreso.

Por otra parte, el cambio radical es la explotación de la tecnología para crear nuevos productos, procesos o servicios que revolucionan un mercado o industria. Además, Gutiérrez, Torres, Jacob, (2001) señalan que el cambio radical se da cuando existe un rompimiento total de lo establecido en un producto o proceso, creando incluso nuevos mercados. El cambio radical se implementa ya sea por una crisis o por una oportunidad.

Una crisis con cierto nivel de magnitud puede afectar el desempeño de una organización. Por otro lado, la organización puede ser consciente de que existe una oportunidad potencial en el mercado, por lo que actúa rápidamente para obtener beneficio antes que sus competidores lo hagan (Price, 2009). El cambio radical se implementa cuando es necesario para interrumpir las formas habituales de hacer las cosas. En este tipo de cambio, el tiempo es vital y las empresas deben actuar con rapidez. La innovación radical ofrece mejoras significativas en el rendimiento y el costo; además, ofrecen nuevas formas de hacer negocios. 
Tipos de innovación incremental y radica

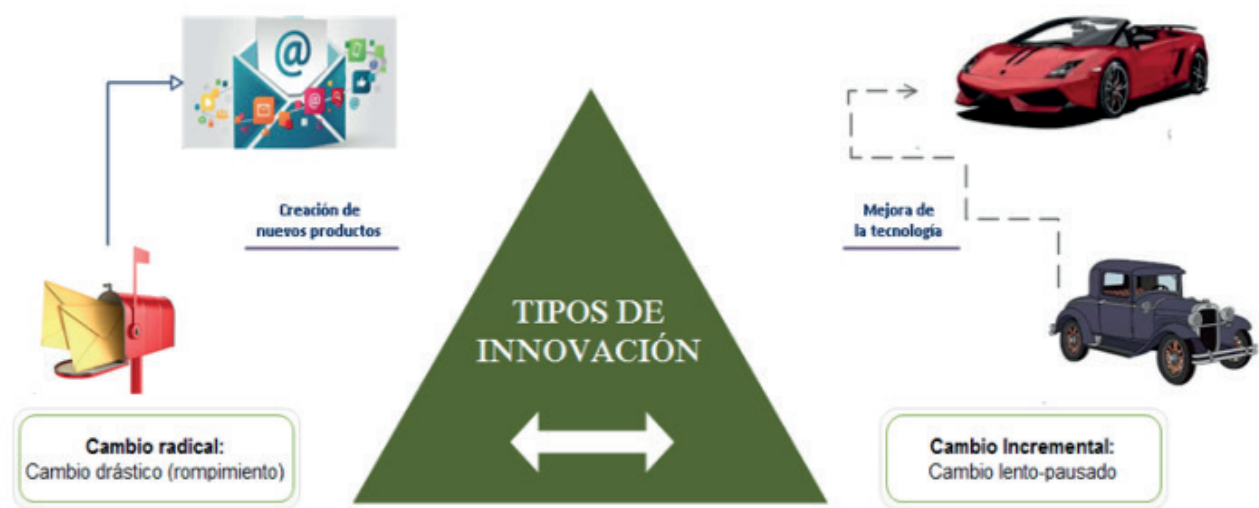

Fuente: Marín-García, J.; Pardo, M; Bonavia, T. (2008); Price, D., 2009, p. 13; James March, 1991, en Leifer et al, 2000 Autor: Por el autor

Por ejemplo, Kodak, que ha dominado el mercado de la fotografía de consumo durante más de un siglo, se encontró en un gran problema a principios de este milenio, cuando se eliminó la película en favor de la imagen digital. La empresa, basada en la mejora incremental de la tecnología anterior, tuvo que enfrentar a empresas nuevas que trajeron consigo la nueva generación de toma de fotografías (Innovación radical). Algunas empresas se muestran reacias a ser radicales e invertir en proyectos largos con un alto nivel de riesgo. Siempre están bajo presión para maximizar el proyecto financiero a corto plazo, y luego, prefieren desarrollar mejoras incrementales de los productos y tecnologías existentes (Leifer et. al, 2000, p. 3)

La empresa Kodak invirtió en cambios incrementales, lo que mantuvo a la organización dominando el mercado fotográfico durante muchos años. Pero cuando los nuevos participantes ingresaron a la industria fotografía con algo completamente innovador, usando la tecnología digital y recursos, pusieron en peligro a la multinacional. Esta no estaba preparada para competir contra ellos ni contra una innovación tan radical. Es así como la empresa que dominó el mercado fotográfico y que siempre se centró en el conocimiento, recursos exis- tentes, y en los desarrollos incrementales, fue fácilmente derrotada. No solo Kodak sino todas las grandes organizaciones están siempre bajo la responsabilidad y presión de adaptarse inmediatamente a la nueva tecnología, cambiar su modelo de negocios, su estructura organizacional, asignación de recursos e inclusive su cultura corporativa con el objetivo de poder sobrevivir y generar riqueza (Yin, Ansari, y Akhtar, 2017).

De acuerdo con el siguiente gráfico, se representa la evolución de las cámaras con el propósito de brindar al lector una mejor comprensión del cambio radical e incremental, determinando las diferencias en el desarrollo de algunas mejoras del conocimiento y los recursos existentes, y el poder de ser completamente radicales, con nuevos conocimientos y recursos.

Es importante señalar que las empresas, según el tipo de innovación, que enfrenten deberán utilizar el mejor enfoque para gestionar el cambio. Dunphy y Stace (1993, en Burne, 2009, p. 349), argumentaron que existen diferentes enfoques, en diversas circunstancias, para gestionar el cambio. Los gerentes y consultores deben saber qué modelo de cambio es el más apropiado de acuerdo con la situación o a la contingencia que se enfrenten. 
Imagen 1. Innovación Incremental y radical

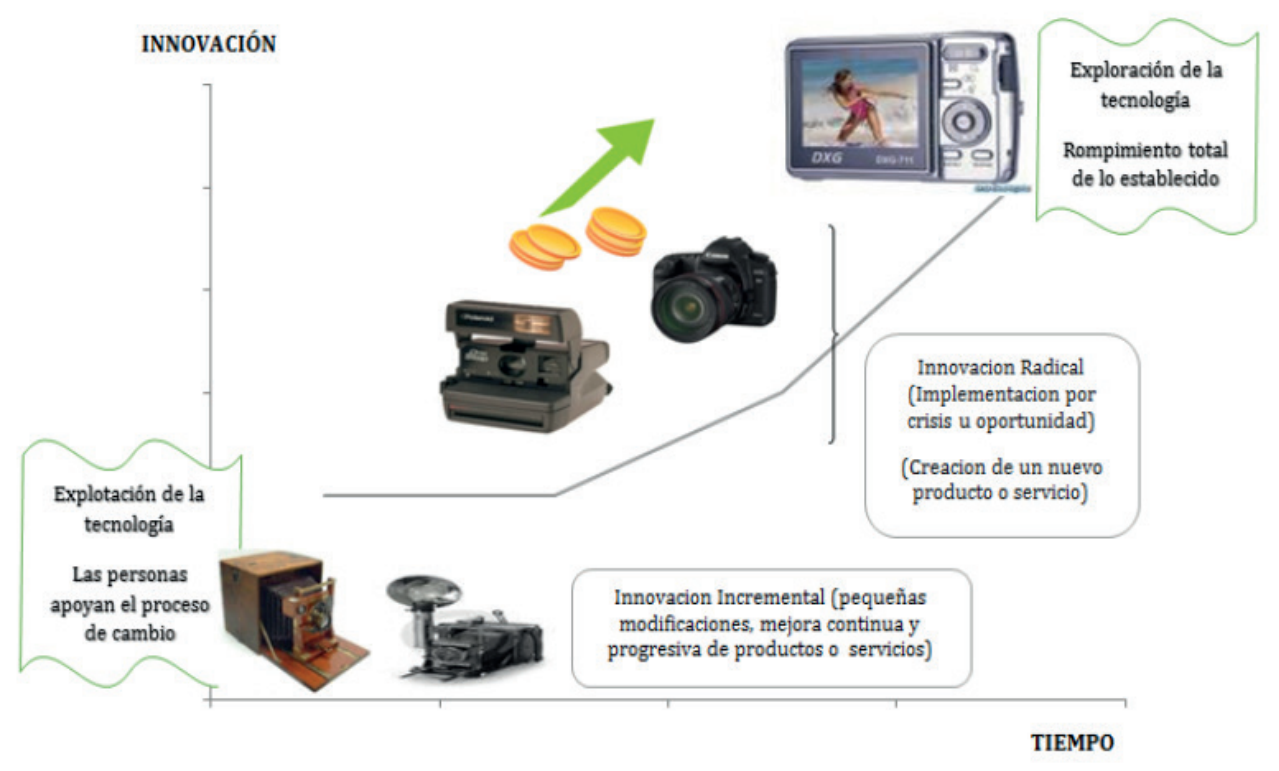

Fuente: Gutiérrez, E; Torres, X; Jacob, M (2001), Price, D., (2009).

\subsection{Proceso de cambio}

Todo cambia a nuestro alrededor y es común que nos resistamos a estos cambios, porque siempre estamos buscando estabilidad y consistencia, pero debemos tener conciencia que, en nuestro entorno, las reglas de juego en la industria seguirán cambiando y debemos estar preparados para enfrentarlas. (Stieglitz, 2006, p.3).

De acuerdo con el modelo del psicólogo Kurt Lewin (en Bush C. y Blake I., 2009, p.9), el proceso de cambio involucra tres etapas diferentes que son necesarias para un cambio efectivo dentro de una organización (ver:

\section{Modelo Lewin. Cambios en la organización}

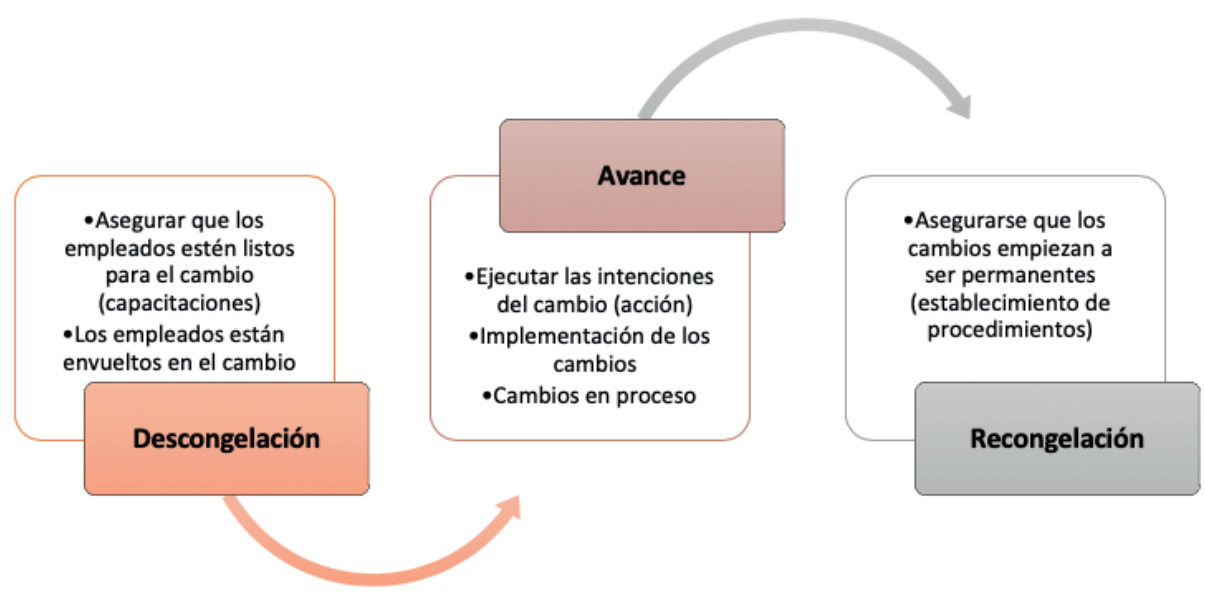

Fuente: Kurt Lewin en Blake, Bush (2009), Hussain, Akram, Lei, Akram, Haider Hussain, S and Ali, M (2016). 
En resumen, la figura indica que las tres etapas son:

- Descongelación. Esta etapa es considerada la fase más difícil en el proceso de cambio, pues implica el movimiento de comprender que el cambio es necesario. Para ello, las organizaciones deben capacitar a todos los colaboradores para que estén conscientes, motivados y listos para el cambio.

- Avance. Es la etapa de transición y es en donde se toma las acciones que generan los cambios que sean necesarios para alcázar competitividad. Estas acciones pueden ocasionar un cambio estructural en las organizaciones, nuevos comportamientos, actitudes y creencias que en conjunto sirven para alcanzar la nueva posición deseada.
Aquí las personas están aprendiendo y desarrollando sus propias soluciones para ayudar en el proceso de cambio.

- Recongelación. En esta etapa es en donde las personas se aseguran de que el cambio se convierta en permanente. Los procedimientos que se solían hacer forman parte del pasado. En el presente es necesario seguir las nuevas formas de trabajar en la organización para lograr el objetivo deseado. (Blake y Bush, 2009).

El proceso de cambio se origina debido a la innovación que viene acompañada de cuatro factores que impulsan el cambio en el mercado. Para poder entender estos factores es importante analizar el modelo de seguimiento realizado por Sheth y Ram, (1987) y que se observa a continuación.

Modelo de seguimiento o fuerzas que impulsan la necesidad de innovar de Sheth y Ram (1987) Modelo de Sheth y Ram en Mitchel, R y Goffin, K (2005, p3). Autor: Sheth y Ram (1987).

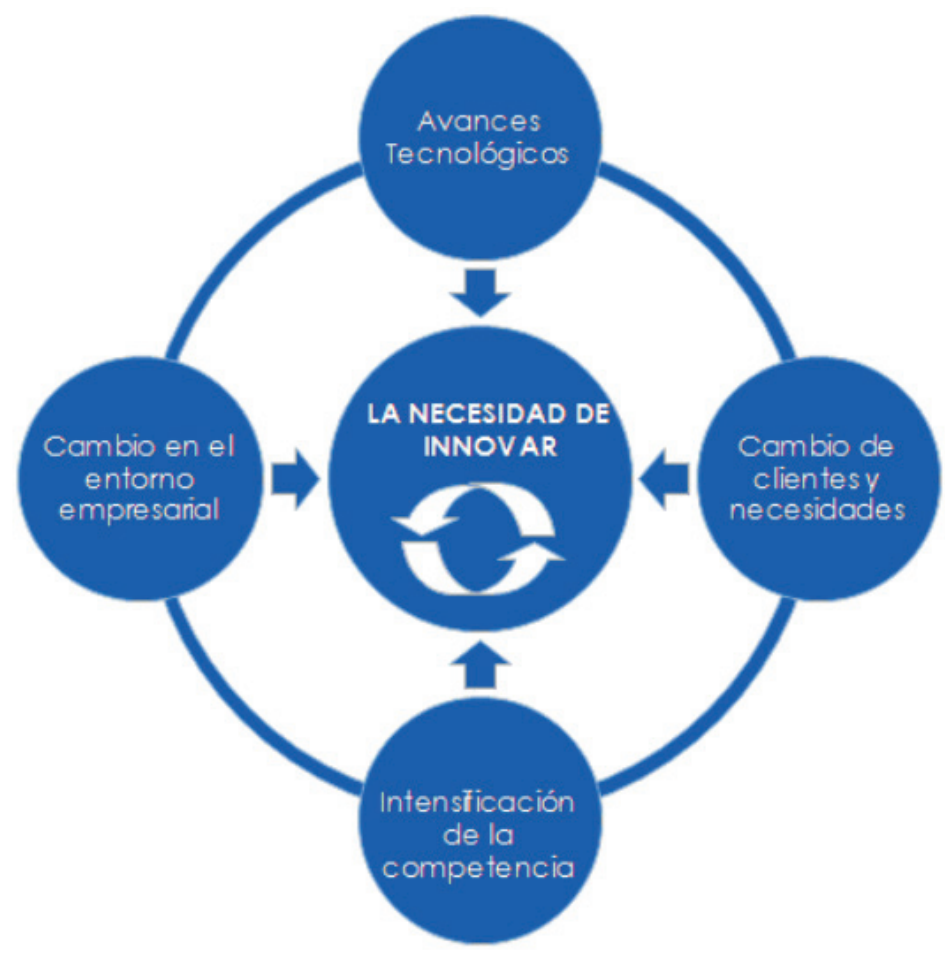


- Avances tecnológicos: Las empresas necesitan invertir en tecnología para poder transformar potencialmente sus mercados. No pueden desarrollarse usando solo recursos internos. Es esencial hacer un seguimiento del progreso de una variedad de tecnologías, debido a su importancia e influencia en los mercados.

- Cambio de clientes y necesidades: Hoy en día, el nuevo escenario de los negocios ha formado un consumidor que demanda mayor calidad y precios más asequibles (HCL, 2009). Por lo tanto, es esencial que las empresas entiendan lo que sus consumidores quieren y necesitan para que no se los lleve la competencia.

- Intensificación de la competencia: Actualmente, la competencia en el mercado obliga a las empresas a encontrar fuentes que les permitan ser innovadores, reducir costos y mejorar la calidad.

Por ejemplo, Guinness tenía una posición excelente en el mercado de acciones de Inglaterra. Con la eliminación de las barreras al comercio internacional (globalización) muchas cervezas nuevas habían llegado a este mercado. Para sobrevivir en su nuevo entorno, la estrategia de Guinness fue invertir en investigación y desarrollo (I+D). Esta empresa trabajó primero para mejorar sus pruebas de calidad, debían asegurarse en vender la mejor versión de su cerveza y cumplir con las exigencias del mercado. Además, buscaba crear un envase completamente nuevo que le permitiría mantener a sus clientes e incluso aumentar nuevos compradores.

Es así que se crea el innovador "In can system". Este sistema consiste en colocar dentro de la lata de cerveza una bolita blanca que hace liberar el gas cuando se la abre y crear la espuma que tanto gusta a los consumidores y que se asemeja a la espuma que se forma cuando se sirve la cerveza directamente de un barril. Esta innovación tuvo un gran éxito ya que Guinness pudo extender el ciclo de vida de su producto, obtener alta rentabilidad, y llevar la tradicional espuma del barril a la lata. Guinness, protegió su invento mediante el uso de patentes (Trott, 2002).

- Cambio de entorno empresarial: El entorno empresarial cambia todo el tiempo. El ambiente de trabajo de hoy no es el mismo de la experiencia de nuestros padres y no será el mismo para nuestras generaciones futuras. Es necesario ser receptivo al cambio según el entorno. Las empresas, al igual que en el pasado, enfrentan revoluciones en su entorno; primero fue la Revolución Industrial y hoy en día su nuevo reto es ser parte de la revolución del internet, las computadoras y la comunicación.

Citando a Krippendorff $(2008$, p. 2) indica que a lo largo de la historia, la civilización con un alto nivel de innovación ha dominado a sus rivales menos creativos. Las sociedades que adoptan nuevas innovaciones siempre obtienen poder sobre sus vecinos y tienen un mejor desarrollo en la agricultura, la metalurgia, la política y en la economía. La vida y la muerte, ganar y perder, el dominio y la sumisión se relacionan con la innovación mientras la humanidad se haya preocupado por tales cosas. La innovación es un factor esencial de supervivencia, no una opción.

Todos estos factores a continuación influyen en las organizaciones sobre la necesidad de innovar.

La innovación lleva a cabo nuevas ideas y valores. La innovación nos permite tener ventajas competitivas y enfrentar las nuevas reglas en el mundo empresarial con éxito. La innovación aporta al entorno em- 
presarial nuevas formas de sobrevivir en el mercado (Rushkoff, 2005, pp. 1-20).

\section{Caso de estudio. Válvulas del Pacifico Pacifvals S.A.}

Válvulas del Pacifico Pacifvals S. A. es una empresa ecuatoriana que se formó en el año 2010 contado con infraestructura, equipos y personal calificado que le permitió desarrollarse en el campo de la industria petrolera, especializándose en la fabricación de cabezales para pozo (Wellhead), árboles de navidad. Sus productos han sido venidos a Petroamazonas EP, GE, Schlumberger, entre otras. En la actualidad, la empresa cuenta con cinco certificaciones internacionales, una de ellas es el API6a que es otorgado por el Instituto de Petróleo americano, que le han permitido incrementar sus productos y servicios. Además, cuenta con el certificado ISO 14001 referente al sistema de gestión ambiental ya que una de sus políticas es fomentar en las operaciones la prevención de la contaminación y la protección ambiental.

Esta investigación determinó el cambio que ha tenido la empresa en estos 9 años de funcionamiento. El análisis está dividido en las variables que utilizó la compañía para poder llegar a resultados que le permitan seguir vendiendo y consolidarse en la industria petrolera, de acuerdo con la siguiente cronología.

Válvulas del Pacifico Pacifvals S. A. y su innovación

\begin{tabular}{|c|c|c|c|c|}
\hline Variable & Estrategias & Mecanismos & Observaciones & Resultados \\
\hline $\begin{array}{l}\text { Formaliza- } \\
\text { ción de los } \\
\text { nuevos ob- } \\
\text { jetivos de } \\
\text { la empresa }\end{array}$ & $\begin{array}{l}\text { Obtener certifica- } \\
\text { ciones internacio- } \\
\text { nales (La organi- } \\
\text { zación empieza } \\
\text { con certificacio- } \\
\text { nes desde el año } \\
\text { 2011) }\end{array}$ & $\begin{array}{l}\text { Cada departamento } \\
\text { se empodera para } \\
\text { estructurar procesos } \\
\text { y cumplir con las exi- } \\
\text { gencias que conlleva } \\
\text { una certificación } \\
\text { Se conforma un equi- } \\
\text { po para auditar cada } \\
\text { proceso, conformado } \\
\text { por una persona de } \\
\text { los diferentes depar- } \\
\text { tamentos: Ingeniería, } \\
\text { Diseño y Desarrollo, } \\
\text { Recursos Humanos Y } \\
\text { SGI (Sistema Integra- } \\
\text { do de Gestión) }\end{array}$ & $\begin{array}{l}\text { La empresa re- } \\
\text { conoce que para } \\
\text { poder subsistir } \\
\text { necesita imple- } \\
\text { mentar procedi- } \\
\text { mientos y adquirir } \\
\text { certificados } \\
\text { internacionales. }\end{array}$ & $\begin{array}{l}\text { 2011: API Q1 (Sistema de } \\
\text { Gestión para la fabricación de } \\
\text { Equipo Petrolero, Petroquími- } \\
\text { ca y Gas) } \\
\text { IS0 9001:2008 (Sistema de } \\
\text { Gestión de calidad) } \\
\text { 2012: API 6A Licencia No. 6a- } \\
\text { 1356 (Licencia para Fabrica- } \\
\text { ción de Cabezales de Pozo, } \\
\text { Árboles de Navidad y Válvulas) } \\
\text { OSHAS 18001: } 2007 \text { (Sistema } \\
\text { de Seguridad y Salud Laboral) } \\
\text { ISO 14001:2008 (Sistema de } \\
\text { Gestión Ambiental) }\end{array}$ \\
\hline $\begin{array}{l}\text { Establecer } \\
\text { programas } \\
\text { de capa- } \\
\text { citación } \\
\text { continua }\end{array}$ & $\begin{array}{l}\text { Capacitar a los } \\
\text { empleados }\end{array}$ & $\begin{array}{l}\text { La persona encar- } \\
\text { gada del Sistema } \\
\text { Integrado de Gestión } \\
\text { es la persona en } \\
\text { guiar e instruir al } \\
\text { personal de Válvulas } \\
\text { del Pacifico }\end{array}$ & $\begin{array}{l}\text { Se envía a capa- } \\
\text { citación externa } \\
\text { a dos empleados } \\
\text { de Válvulas del } \\
\text { Pacifico Pacifvals } \\
\text { S.A para fortale- } \\
\text { cer el sistema de } \\
\text { calidad }\end{array}$ & $\begin{array}{l}\text { La organización identifica } \\
\text { cuellos de botella en la toma } \\
\text { de decisiones y procesos } \\
\text { operativos }\end{array}$ \\
\hline
\end{tabular}




\begin{tabular}{|c|c|c|c|c|}
\hline Variable & Estrategias & Mecanismos & Observaciones & Resultados \\
\hline $\begin{array}{l}\text { Mantener } \\
\text { el compro- } \\
\text { miso de la } \\
\text { dirección }\end{array}$ & $\begin{array}{l}\text { Fortalecer la } \\
\text { cultura, visión } \\
\text { y misión de la } \\
\text { compañía }\end{array}$ & $\begin{array}{l}\text { Dar apoyo al siste- } \\
\text { ma de gestión de } \\
\text { calidad (reuniones } \\
\text { continuas) }\end{array}$ & $\begin{array}{l}\text { Se realiza trabajos } \\
\text { en grupos para } \\
\text { que cada de- } \\
\text { partamento se } \\
\text { familiarice con el } \\
\text { rol de cada área } \\
\text { para alcanzar los } \\
\text { objetivos }\end{array}$ & $\begin{array}{l}\text { La gerencia acepta subgeren- } \\
\text { cias emitidas por los emplea- } \\
\text { dos para avanzar en la mejora } \\
\text { continua }\end{array}$ \\
\hline $\begin{array}{l}\text { Fomentar } \\
\text { y aplicar } \\
\text { innovación }\end{array}$ & $\begin{array}{l}\text { Analizar las } \\
\text { necesidades de } \\
\text { los clientes, de } \\
\text { los empleados } \\
\text { y del mercado } \\
\text { de la industria } \\
\text { petrolera }\end{array}$ & $\begin{array}{l}\text { Estudio de costo } \\
\text { y beneficio de las } \\
\text { nuevas implementa- } \\
\text { ciones en tres ejes } \\
\text { fundamentales de la } \\
\text { empresa los cuales } \\
\text { son: } \\
\text { Mejoramiento de los } \\
\text { productos (Innova- } \\
\text { ción Incremental), } \\
\text { Creación de nuevos } \\
\text { productos y servi- } \\
\text { cios, e } \\
\text { Implementación y } \\
\text { mejora de nuevos } \\
\text { procedimientos que } \\
\text { faciliten la toma de } \\
\text { decisiones }\end{array}$ & $\begin{array}{l}\text { Se identifica la } \\
\text { necesidad de } \\
\text { mejorar los pro- } \\
\text { ductos, ampliar el } \\
\text { portafolio, mejorar } \\
\text { la planificación } \\
\text { de recursos y } \\
\text { reestructurar } \\
\text { la organización } \\
\text { para la toma } \\
\text { de decisiones y } \\
\text { responsabilidades }\end{array}$ & $\begin{array}{l}\text { 2014: Los departamentos de } \\
\text { Ventas, Producción. Compras } \\
\text { y Financiero pasan de utilizar } \\
\text { Excel al sistema SAP Bussi- } \\
\text { ness One (Sistema de planifi- } \\
\text { cación de recursos ERP) } \\
\text { 2016: Nueva restructuración, } \\
\text { se reafirma la Gerencia Gene- } \\
\text { ral y se crea } 2 \text { coordinaciones: } \\
\text { Financiera y Operaciones con } \\
\text { el objetivo de cambiar de una } \\
\text { organización vertical a una } \\
\text { horizontal. } \\
\text { 2017: La compañía inicia el re- } \\
\text { gistro de } 4 \text { patentes en el Ins- } \\
\text { tituto Ecuatoriano de Propie- } \\
\text { dad Intelectual (IEPI) referente } \\
\text { a sus nuevos productos } \\
\text { 2016: La empresa ofrece ser- } \\
\text { vicio de reparación y reacondi- } \\
\text { cionamiento de pozos } \\
\text { 2018: Packers y flow control. }\end{array}$ \\
\hline
\end{tabular}

La empresa para tener rentabilidad cambio y fue creativa. Primero se aseguró de que los empleados conozcan sobre las diferentes transformaciones que iba a realizar la compañía (formalización de los objetivos), y brindo el apoyo a cada departamento para que se empodere y elabore los nuevos procesos. De igual manera, aplicó mecanismos para ejecutar los cambios, mantenerlos y obtener los resultados deseados (Obtención de certificaciones internacionales). También la compañía capacitó a su personal tanto internamente como externamente para fortalecer los cambios. Es en este punto donde se identifica problemas en la toma de de- cisiones y procesos operativos. La empresa acepta las sugerencias emitidas por los empleados, creando trabajos en grupos para que cada departamento entienda su rol en el proceso y como su desempeño afecta a las áreas. Finalmente, Válvulas del Pacifico Pacifvals S. A. toma la decisión de integrar a todos los departamentos y pasa a manejar las operaciones de Excel a un sistema integral de planificación de recursos (ERP) llamado SAP Bussines One. Cada jefe/líder de área diseño el sistema de acuerdo con sus necesidades y procedimientos. La segunda decisión de la empresa fue reafirmar el compromiso de la Gerencia General para fortalecer la cultura, 
misión y visión de la organización, se crea las coordinaciones financieras y de operaciones para que trabajen muy de cerca con todos los departamentos y de esta manera poder agilizar la toma de decisiones y pasar de una estructura vertical a una horizontal.

La estructura vertical es aquella que está altamente jerarquizada, con muchos mandos medios y dispone lo que deben hacer, limitando tanto la visión y los objetivos de las empresas, al igual que dificulta la coordinación con otras áreas; por otra parte, la estructura horizontal permite una relación directa entre los trabajadores y cada uno de los departamentos (integración). La empresa tiene un diseño más plano y menos mandos medios (Valenzuela, 2013).

En cuanto, a la innovación de productos y servicios, la empresa identificó un cambio en el entorno empresarial. La industria del petróleo sufrió una baja del precio del crudo en más del 50\% lo que afectó directamente el presupuesto general del Estado, estancando la economía ecuatoriana y abrió la puerta a la recesión (España, 2015). Esto sumado a la globalización de mercados donde se intensifica la competencia más que nunca antes, empujó a la compañía a mejorar sus productos (Innovación Incremental) y aumentar sus productos y servicios para poder cumplir con las necesidades de sus clientes, de su entorno y así poder sobrevivir y seguir siendo rentable en su industria.

\section{Conclusión}

La innovación nace en la creación de nuevos productos o servicios e incluso puede originar la creación de nuevos mercados y modificación de la estructura de las empresas. La innovación crea nuevos negocios altamente rentables ya que logra ventaja competitiva sobre sus competidores y llena las necesidades de los clientes de una manera ciertamente mejor.
Existen dos tipos de innovación: incremental y radical. La primera utiliza la tecnología ya existente para progresivamente mejorar los productos o servicios existentes (mejora continua). En este tipo de innovación los empleados están preparados y capacitados para apoyar y contribuir en los procesos de cambio que inician las organizaciones. En cambio, la innovación radical desarrolla nueva tecnología creando nuevos productos o servicios que originan una ruptura entre normas establecidas que afectan directamente el desempeño de las organizaciones. Por lo tanto, es importante que las empresas realicen una estrategia adecuada de cambio que permita enfrentar con éxitos los cambios. Las organizaciones que no actúen con rapidez corren el riesgo de desaparecer.

Las organizaciones necesitan cambiar según el mercado en el que desarrollan sus actividades y para estar listas al cambio, necesitan que sus empleados entiendan y estén listos para la trasformación. Deben capacitarlos, guiarlos y ejecutar las acciones necesarias para avanzar. Las empresas necesitan asegurarse de que los cambios sean permanentes con el establecimiento de procedimientos.

Las empresas necesitan innovarse, ya que siempre enfrentan cambios en su entorno, la tecnología evoluciona, se intensifica la competencia y las necesidades de los clientes sufren cambios que hay que llenar. Por lo que, innovarse es el único camino para seguir generando ventas y permanecer posicionado en la mente del consumidor.

La empresa Válvulas del Pacifico Pacifvals S. A. entendió la coyuntura que vive la industria petrolera en el Ecuador y la necesidad de acoplarse a su nuevo entorno para poder crear una ventaja competitiva que le permita seguir en el mercado. Es así que la organización empieza el cambio estructural formalizando los nuevos objetivos de la empresa y brindado un total apoyo a los emplea- 
dos para la elaboración de procedimientos que les permitan no solo asegurar cambios sino también obtener certificaciones internacionales e identificar de mejor manera los problemas que enfrentaba la organización. De igual manera, fortalece la cultura organizacional, la misión y la visión de la empresa aceptando sugerencias de sus empleados.

La empresa Válvulas del Pacifico Pacifvals S. A. identifica la necesidad de innovar los productos ya existentes y de ampliar su portafolio. De igual manera busca mejorar el manejo de sus recursos integrando a todos los departamentos y reestructurando la organización a una estructura horizontal que permita facilitar la toma de decisiones y responsabilidades.

Las innovaciones utilizadas tanto en sus productos como en sus procesos y métodos de gestión (estructura organizativa) han permitido a esta empresa seguir desarrollándose en su industria, optimizando sus costos de producción, agilizando la toma de decisiones y llenando las expectativas de sus clientes.

\section{Referencias bibliográficas}

Amit, R; Zott, C. (2010). Business Model Innovation: Creating Value in Times of Change Working Paper 870, IESE Business School, University of Navarra, 17 pages

Blake, I; Bush, C. (2009). Project managing Change: Practical Tools ans Techniques to Make Happen, Finacial Times, Series, ISBN 9780273-72045-4, Unit5ed Kingdom, Pearson Education Limited

Burnes, B. (2009). Managing Change, Fifth edition, London: Prentice Hall.

Burns, P. (2001). Entrepreneurship and Small Business, Macmillan Press Ltd, China.

Dobson et. al. (2004). Strategic Management, Blackwell Publishers Ltd, USA.

Drucker, P. (1985). Innovation and Entrepreneurship, Richard Clay Ltd, Great Britain.

España, S. (19/09/2015). La caída del petróleo acerca a Ecuador a la recesión. El Presi- dente del país. Rafael Correa admite que la economía ecuatoriana se está estancando. Diario el País. Obtenida de: https:// elpais.com/internacional/2015/09/19/ actualidad/1442615187_094855.html

Marín-Garcia, J.; Pardo, M.; Bonavia, T. (2008). La mejora continua como innovación incremental: El caso de una empresa industrial española. Economía Industrial, No. 368, pp. 155-167

Gutiérrez, E.; Torres, X.; Jacob, M. (2001). Innovación tecnológica: ideas básicas, III edición COTEC, 2001

HCL (2009). Global Product Life Cycle Management, Improving product profitability amidst global competition through global sourcing. Obtenido de: file:///E:/PAPERS/ global-product-life-cycle-management. pdf

Hussain, S.; Akram, S.; Lei, S.; Akram, T.; Haider, M.; Hussain, S and Ali, M. (2016). Kurt Lewin's process model for organizational change: The Role of leadership and employee involvement: A critical Review. Journal of Innovation \& Knowledge, pp 1-7, JIK 26

Krippendorff, K. (2008). The Way of Innovation, Platinum Press

Leifer, R. et. al. (2000). Radical Innovation: How mature companies can outsmart upstarts, Harvard Business School Press.

Lutolf, C. (2009). From Innovation to Cash Flows: Value Creation by Structuring High-Technology Alliances, New Jersey: Wiley.

Mitchell, R. and Goffin, K. (2005). Innovation Management: Strategy and Implementation using the pentathlon framework, Palgrave, Macmillan.

Palmer \& Hartley. (2006). The Business Environment, McGraw-Hill Companies, Inc, UK

Price, D. (2009). The principles and practice of change, Palgrave Macmillan, Business school.

Rushkoff, D. (2005). Get Back in the Box, Innovation from the Inside Out, HarperCollins Publisher, USA.

Stieglitz, D. (2006). Taming the Dragons of Change: 10 Tips of Achieving Happiness and Success When Everything Around You is Changing, PhD. 
Sydie R, A. and Adams B. (2001). Sociological Theory, United States: Pine Forge Press

Shumpeter, J. (1934). The Theory of Economic Development, Cambridge, MA: Harvard University Press.

Schumpeter, J. A. (1911). The Theory of Economic Development, Harvard University Press, Cambridge, MA.

Sundbo, J. (1998). The Theory of Innovation: Entrepreneurs, technology and Strategy, Edward Elgard.

Trott, P. (2002). Innovation Management and New Product Development, Person Education Limited, England.
Valenzuela, J (2013). La Evolución del Diseño y la Estructura Organizativa: Un estudio de Casos. Grado de Relaciones Laborales, Universidad Autónoma Barcelona, España. Obtenida de: https://ddd.uab.cat/pub/tfg/2013/109361/ TFG.jvalenzuelasevilla.pdf

Wickham, P. (2006). Strategic Entrepreneurship, Fourth edition, Practice Hall.

Yin, E.; Ansari, S.; Akhtar, N. (2017). Radical Innovation, Paradigm Shift and Incumbent's Dilemma The case of the Auto Industry. Future Studies Research Journal: Trends and Strategies. Sao Paulo, V.9, N1, p. 138148 Jan./ Apr. 